Check for updates

Cite this: RSC Adv., 2018, 8, 7932

Received 3rd October 2017

Accepted 2nd February 2018

DOI: 10.1039/c7ra10916d

rsc.li/rsc-advances

\section{Design of electret polypropylene melt blown air filtration material containing nucleating agent for effective PM2.5 capture $\uparrow$}

\begin{abstract}
Haifeng Zhang, (D) Jinxin Liu, (D) Xing Zhang, Chen Huang (iD) and Xiangyu Jin*
Particulate matter (PM) pollution has become a serious threat to public health, climate and ecosystems. Therefore, filtration materials with high filtration efficiency and low air resistance are in urgent need. Electret filters that can significantly improve filtration efficiency without increasing pressure drop are widely used for air filtration. However, long-term maintenance of the electrical charges on electret filters, particularly at high temperatures and in wet conditions, is still a challenge. Herein, we report the fabrication of a novel electret filter with a fluffy structure, high porosity and satisfactory filtration stability using polypropylene (PP) as the matrix polymer and magnesium stearate (MgSt) as the charge enhancer. Benefiting from the fluffy structure created by melt blown at a large die-to-collector distance (DCD), the pressure drop decreased from 67.0 Pa to $52.9 \mathrm{~Pa}$ at an air flow rate of $85 \mathrm{~L} \mathrm{~min}^{-1}$. Furthermore, the introduction of MgSt changed the crystal structure of the melt blown nonwovens, thus improving the electret properties to achieve a high filtration efficiency of $99.22 \%$, a low pressure drop of $92 \mathrm{~Pa}$ and a satisfactory QF of $0.054 \mathrm{~Pa}^{-1}$. In particular, the electret filtration materials exhibited excellent filtration stability and loading performance. Thus, this electret material may become a good candidate for personal protective filtration materials against PM2.5.
\end{abstract}

\section{Introduction}

In recent years, fine particulate matter (PM) pollution has drawn worldwide attention due to its severe threat to public health, climate and ecosystems. ${ }^{1,2}$ These fibrous filters have been widely used for PM filtration owing to their beneficial features such as energy-efficiency, cost-effectiveness and ease of scalable fabrication. ${ }^{3,4}$ In general, fibrous filters can be classified as spunbonded filters, needle-punched filters, melt blown filters and electrospun filters. Among them, traditional filters, such as spun-bonded filters and needle-punched filters, capture the particles primarily by a mechanical filtration mechanism. Thus, there is the drawback that it is difficult to achieve high filtration efficiency because of the large fiber diameter and pore size., ${ }^{5,6}$ Although the filtration efficiency can be enhanced by increasing the basis weight accompanied with a high-pressure drop, this step would cause a low quality factor. In addition, electrospun filters have gained growing attention attributed to the large surface to volume of these nanofibers, which achieve high filtration efficiency. ${ }^{7}$ However, some disadvantages restrict the development of electrospun filters, such as the high pressure drop and the difficulty in industrializing their manufacture. ${ }^{8}$

Engineering Research Center of Technical Textiles, Ministry of Education, College of Textiles, Donghua University, No. 2999 North Renmin Road, Songjiang, Shanghai 201620, China. E-mail: jinxy@dhu.edu.cn

$\dagger$ Electronic supplementary information (ESI) available. See DOI: 10.1039/c7ra10916d
Presently, melt blown nonwovens are the mainstream fibrous air filters, because of their high surface area per unit weight, high porosity, tight pore size and high barrier properties. ${ }^{9,10}$ In particular, benefiting from the capacities of storing abundant charges and creating a quasi-permanent electric field on the periphery of their fibers, electret melt blown filters have been proved to be promising for intensively filtrating PM2.5 by electrostatic force.11,12 However, due to bulk conductivity, surface conductivity and ionic attacks from the surrounding environment, it is inevitable that the charge will decay after a period of time, which results in significant reduction of filtration efficiency. ${ }^{13}$ To date, a handful of efforts have been made to improve their filtration performance by blending additives with the polymer. Yu et al. added tourmaline particles to polylactic acid (PLA) polymer to fabricate melt blown nonwovens and achieved a filtration efficiency of $88 \%$. They attribute the improvement to the increase in friction between the fiber web with the tourmaline particles and collector, which can improve the electrostatic adsorption capacity of the melt blown nonwoven to particles. ${ }^{14}$ Kilic et al. fabricated polypropylene (PP) electret melt blown nonwovens containing barium titanate particles with a filtration efficiency of $99.97 \%$ at a pressure drop of $95 \mathrm{~Pa}$. The reason for their good performance was that barium titanate is a dielectric material that can improve the localized polarizability within the polymer owing to its high dielectric constant. ${ }^{15}$ Brochocka et al. fabricated electret perlite-modified PP melt blown nonwovens with a filtration efficiency of $99.56 \%$ and a pressure drop of $290 \mathrm{~Pa} .{ }^{16}$ Xiao et al. 
added stearate and modified rosin into the PP fabrics during melt blown process and the filtration efficiency reached $99.68 \%$ and $99.43 \%$, respectively. ${ }^{17}$ Significantly, the filtration efficiency could be further improved by introducing additives. However, these electret melt blown nonwovens were still subject to several drawbacks, such as their relatively high filtration resistance. A few researches tackled this problem by optimizing the materials structure. ${ }^{\mathbf{1 8 , 1 9}}$ In addition, the electret stability, in particular, the low filtration stability under high temperature and humidity still limits their application. As a result, fabrication of electret materials for air filtration with low air resistance and excellent charge stability is still a challenge to be resolved.

In this study, we report a facile strategy to fabricate nonwovens via melt blown and corona charging technology, resulting in filtration materials with excellent electret properties, high filtration efficiency and good air penetrability. Inspired by the fact that the nucleating agent could improve the crystal structure and further improve the electret performance, nucleating agents, namely, magnesium stearate (MgSt) particles were added as the charge enhancer during melt blown. Through optimizing the ratio of $\mathrm{MgSt}$, the $\mathrm{PP} / \mathrm{MgSt}$ electret melt blown nonwoven filters was successfully fabricated and its structural properties, filtration performances and the mechanism of the enhanced filtration performance were investigated. The filtration performance of the nonwoven filters, including the loading test performance and the filtration stability at high temperature or humidity, were further studied.

\section{Experimental}

\section{Materials}

PP polymer resin with a melt flow index of $1500 \mathrm{~g}$ (per $10 \mathrm{~min}$ ) was provided by Sinopec Shanghai Petrochemical Co. Ltd. MgSt powders with a melt flow index of $400 \mathrm{~g}$ (per $10 \mathrm{~min}$ ) were purchased from Sinopharm Chemical Reagent Co. Ltd.

\section{Melt blown nonwovens preparation}

The melt blown nonwovens were produced using a melt blown line having 142 spinnerets (diameter $=0.25 \mathrm{~mm}$ ). The air plate angle was $60^{\circ}$. The schematic illustration of the fabrication process of PP melt blown nonwovens and the corona charging technology is shown in Fig. 1. When preparing the melt blown nonwovens, the main parameters are the throughput of polymer, the die to collector distance (DCD), the air temperature, the air pressure and the belt velocity. By adjusting these parameters, fiber diameter and structure of the melt blown nonwovens can be controlled. Typical processing parameters are listed in Table 1 and the melt blown nonwovens with different concentration of MgSt powders ( $0 \mathrm{wt} \%, 0.1 \mathrm{wt} \%$, $0.3 \mathrm{wt} \%, 0.5 \mathrm{wt} \%, 0.7 \mathrm{wt} \%$ and $1 \mathrm{wt} \%)$ were produced.

\section{Corona charging}

The corona charging apparatus is composed of a high voltage power supply, a point-shaped electrode and a grounding electrode. Due to the high potential between the high voltage power and the roller-shaped electrode, corona discharge took place,

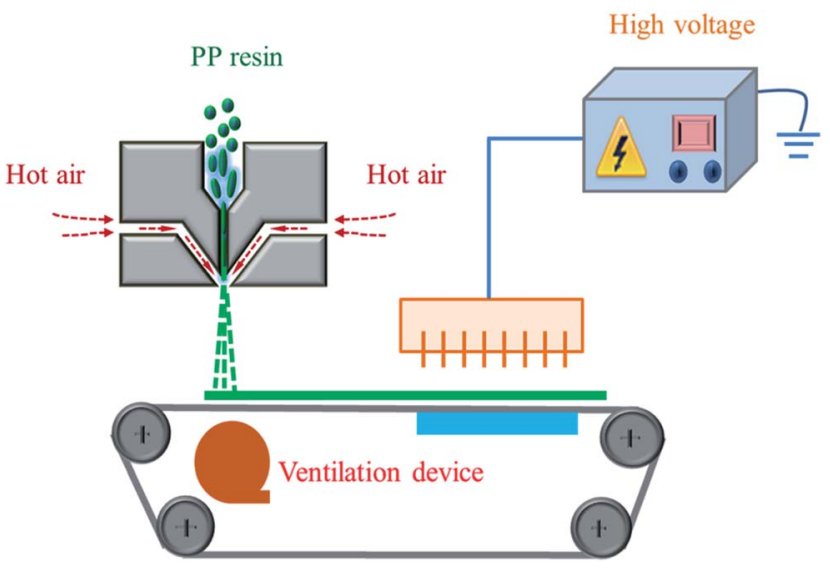

Fig. 1 Schematic illustration of the fabrication procedure of the PP melt blown nonwovens and the corona charging technology.

resulting in deposition of the ions on the electret materials. ${ }^{20}$ In this study, all of the samples were charged at a voltage of $100 \mathrm{kV}$ for $30 \mathrm{~s}$ and the charging distance between electrodes was $100 \mathrm{~mm}$.

\section{Characterization}

The surface morphology of melt blown nonwovens was investigated using a scanning electron microscope (SEM, TM 3000, Hitachi Ltd., Japan HQ). The fiber diameters and their size distribution were calculated using the software Nano Measurer 1.2.5 by measuring 50 fibers from the SEM images. The pore structure of the melt blown nonwovens was investigated using a capillary flow porometer (CFP-1100AI, Porous Materials Inc., USA) based on the bubble point test. The porosity was calculated by the following formula:

$$
P=\left(1-\frac{m}{\rho \delta}\right) \times 100
$$

where $\rho, m$ and $\delta$ represent the fiber density, web density and web thickness, respectively.

The filtration performance of the electret melt blown nonwovens was tested using an automated filter machine (TSI 8130, TSI Instruments Co. Ltd., America). In this study, sodium chloride was used to generate aerosol particles using an atomizing air pump with a mass median diameter of $0.26 \mu \mathrm{m}$. The geometric standard deviation of the particles was less than $1.86 \%$. When testing the filtration performance, the aerosol particles passed through the samples with a valid test area of $100 \mathrm{~cm}^{2}$ at the air flow rate of $85 \mathrm{~L} \mathrm{~min}^{-1}$. Each sample was tested five times to ensure accuracy. The filtration efficiency could be calculated as follows:

$$
\eta=1-\frac{\varepsilon_{1}}{\varepsilon_{2}}
$$

where $\eta$ represents the filtration efficiency, $\varepsilon_{1}$ and $\varepsilon_{2}$ are the quantity of the aerosol particles in the downstream and upstream of the filter, respectively. The pressure drop of the sample was measured by a flow gauge and two electronic pressure transmitters. The detailed information about the test 
Table 1 The processing parameters of melt blown nonwoven filters

\begin{tabular}{|c|c|c|c|c|c|c|c|}
\hline Parameter & $\begin{array}{l}\text { Screw } 1 \text { temperature } \\
\left({ }^{\circ} \mathrm{C}\right)\end{array}$ & $\begin{array}{l}\text { Screw } 2 \text { temperature } \\
\left({ }^{\circ} \mathrm{C}\right)\end{array}$ & $\begin{array}{l}\text { Screw } 3 \text { temperature } \\
\left({ }^{\circ} \mathrm{C}\right)\end{array}$ & $\begin{array}{l}\text { Air temperature } \\
\left({ }^{\circ} \mathrm{C}\right)\end{array}$ & $\begin{array}{l}\text { Screw speed } \\
(\mathrm{rpm})\end{array}$ & $\begin{array}{l}\text { Distance } \\
(\mathrm{mm})\end{array}$ & $\begin{array}{l}\text { Air pressure } \\
(\mathrm{MPa})\end{array}$ \\
\hline & 282 & 341 & 344 & 300 & 2.78 & 100 & 0.25 \\
\hline
\end{tabular}

equipment is shown in Fig. S1. $\dagger$ The test process was carried out at an ambient temperature of $25 \pm 2{ }^{\circ} \mathrm{C}$ and a relative humidity of $45 \pm 5 \%$. The heat treatment experiments were carried out at a high and low temperature test chamber (H/GDWJS-50L, Shanghai Husheng Instruments Co. Ltd., China). The samples were treated at $70{ }^{\circ} \mathrm{C}$ for $24 \mathrm{~h}$.

The surface electrostatic potential was measured using a non-contacting voltmeter (TREK-542A-2-CE, TREK Inc., USA). Wide-angle X-ray diffraction was measured using an X-ray diffractometer (D/max-2550VB $+/ \mathrm{PC}$, Japan) in continuous mode to determine the degree of crystallinity and the crystal structure. The surface electret properties were investigated by thermally stimulated discharge (TSD) techniques. The opencircuit TSD measurements were carried out in a system consisting of a temperature controlled oven with a linear heating rate of $3{ }^{\circ} \mathrm{C} \min ^{-1}$, an electrometer (Model6514, Keithley), and a data processing computer.

\section{Results and discussion}

\section{Design and fabrication of the fluffy melt blown nonwovens}

The web properties. In this study, we aim to design a filtration material with low air resistance and high filtration efficiency. For this purpose, we fabricated the nonwoven filters according to the following criteria: (1) fine fiber diameter and small pore size to achieve a relatively high filtration efficiency, (2) high porosity to allow the easy passage of air, (3) threedimensional (3D) micro porous structure to avoid clogging during long filtration time. These three objectives could be achieved by a facile method of melt blown technology at a large DCD. With the increase in DCD, the high-speed cool air worsened the adhesion between the fibers. Moreover, the collecting time also increased, which was beneficial for forming a fluffy structure. ${ }^{21}$ As a result of this factor, a fluffy three-dimensional micro porous structure melt blown nonwoven filter was acquired. Then, the corona charging technology was applied to further improve the filtration efficiency by electrostatic attraction effects and the additive was also introduced to improve the electret performance.

To create the fluffy and high porosity structure, we fabricated the melt blown nonwovens by regulating the DCD from $10 \mathrm{~cm}$ to $35 \mathrm{~cm}$. As shown in Fig. 2a-f, a typical randomly arranged three-dimensional micro porous structure was prepared, thus making it an appropriate candidate for air filtration. The average diameters are shown in Fig. $2 \mathrm{~g}$ and $\mathrm{S} 2 . \dagger$ No visible change in the fiber diameter is found with the increase in DCD. This was because the fiber drawing process primarily occurred at a close region (about $1.5 \mathrm{~cm}$ from the die) and the hot air rapidly cooled when the DCD exceeded the drawing region. ${ }^{22}$ The pore size, pore size distribution and porosity were further investigated. As shown in Fig. $2 \mathrm{~h}$, the average pore size ranged from $14.5 \mu \mathrm{m}$ to $14.8 \mu \mathrm{m}$, indicating that the pore sizes were also not affected by the DCD. This can be ascribed to the fact that pore sizes are closely related to fiber diameter. The pore size distributions further confirmed the conclusion. All samples presented identical pore distribution peaks at $14.6 \mu \mathrm{m}$ (Fig. 2i), which demonstrated that all the samples possessed uniform and similar pore size distributions. However, the porosity gradually increased with an the increase in DCD because the fibers had more time to cool and it was difficult for them to bond together from the residual heat, thus resulting in the formation of a fluffy structure. ${ }^{23}$ In addition, the "fiber strand" phenomenon occurred with an increase in DCD, primarily because the large fiber drawing region before collection whipped the fibers drastically and entangled them more severely together, ${ }^{24}$ which also increased the fluffiness. The high porosity indicates that air flow would choose to pass through the media in a shorter and unblocked path by obeying the minimal resistance principle. ${ }^{25}$ On this basis, good air permeability could be achieved.

The filtration properties. Considering that the properties of melt blown nonwovens changed with an increase in DCD, we can reasonably speculate that the filtration performances also changed correspondingly. The filtration performances before and after charging were investigated. As shown in Fig. 3a, for the uncharged samples, the filtration efficiencies obtained at various DCD were 52.55\%, 52.02\%, 51.83\%, 51.54\%, 48.95\% and $46.56 \%$, while the corresponding pressure drops were 67.0, 63.5, 56.2, 52.9, 50.5 and $48.1 \mathrm{~Pa}$, respectively. Both the filtration efficiency and the pressure drop of all of the samples synchronously decreased with an increase in DCD. This may be attributed to their fluffy structures with larger porosity (Fig. 3c), which reduces the collision between particles and fibers according to Brownian diffusion, thus resulting in intensified permeability of both air and particles. ${ }^{26}$

The quality factor (QF) is widely used as a comprehensive indicator of filter performance. The QF of a filter is defined as follows:

$$
\mathrm{QF}=\left(\ln \frac{1}{1-\eta}\right) / \Delta p
$$

where $\eta$ represents the filtration efficiency of the aerosol and $\Delta p$ is the pressure drop. ${ }^{27}$ As shown in Fig. 3b, the QF value presents an escalating trend with an increase in DCD from 10 to $25 \mathrm{~cm}$ and achieved a maximum value of $0.0136 \mathrm{~Pa}^{-1}$ at $25 \mathrm{~cm}$. However, as the DCD further increased to $35 \mathrm{~cm}$, the QF displayed a conspicuously decreasing trend. This could be because the filtration efficiency declined slowly with an increase in DCD from 10 to $25 \mathrm{~cm}$, while the pressure drop decreased heavily. Nevertheless, when the DCD increased to $35 \mathrm{~cm}$, the fluffy 

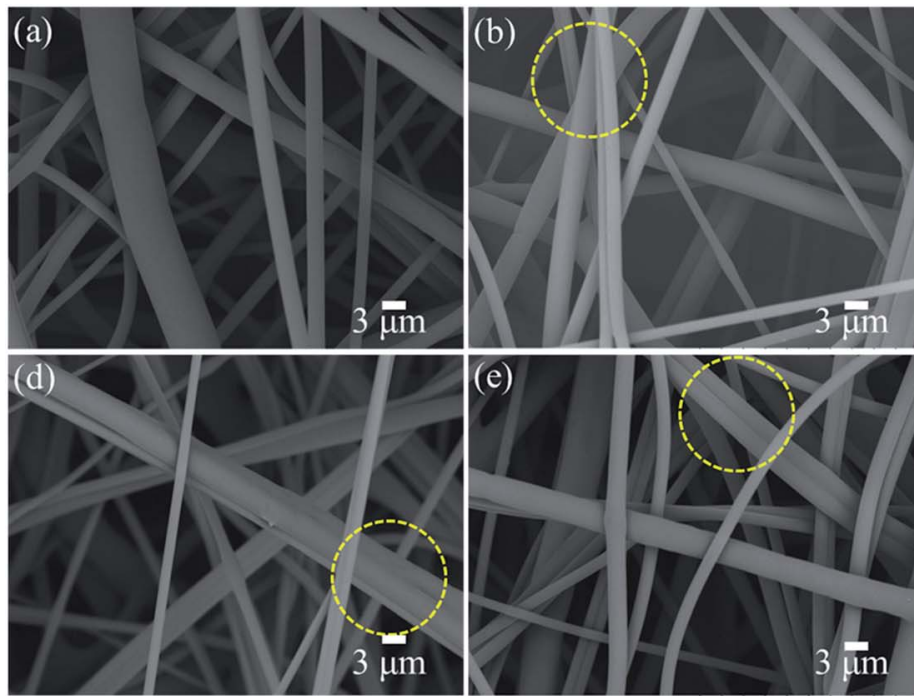

(g)

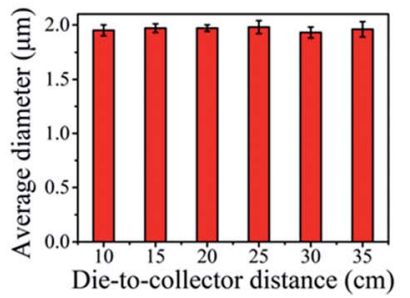

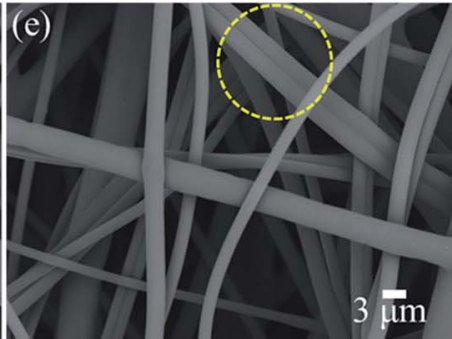

(h)

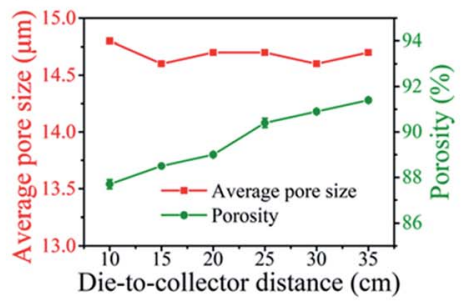

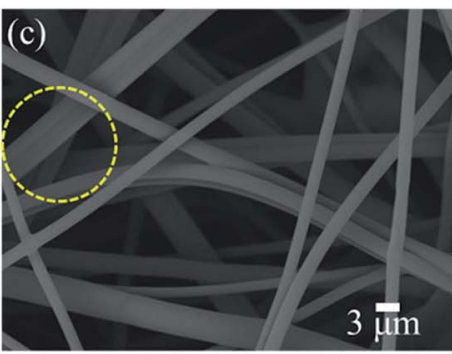

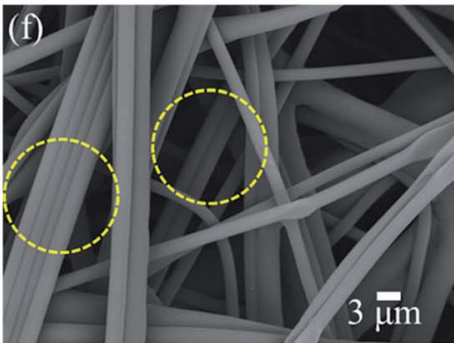

(i)

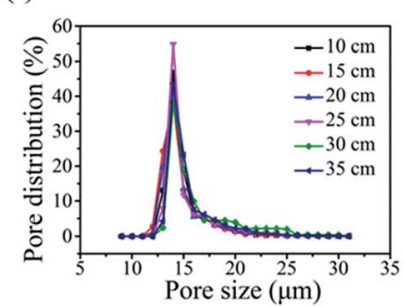

Fig. 2 Morphology and pore structure of PP melt blown nonwovens. SEM images of PP melt blown fabricated at DCD of (a) 10, (b) 15, (c) 20, (d) 25, (e) 30 and (f) $35 \mathrm{~cm}$. (g) Average diameter, (h) average pore size and porosity, (i) pore size distribution.

(a)

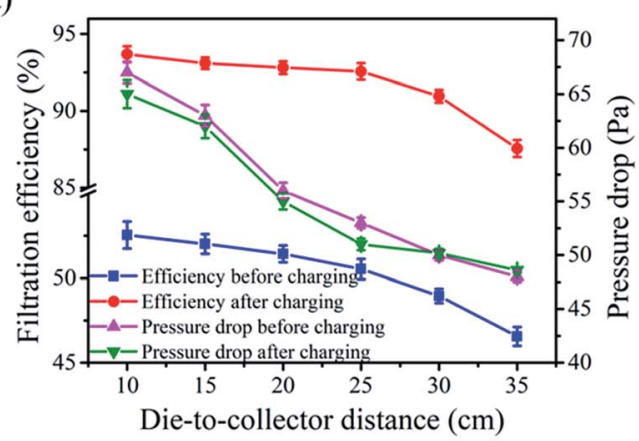

(c)

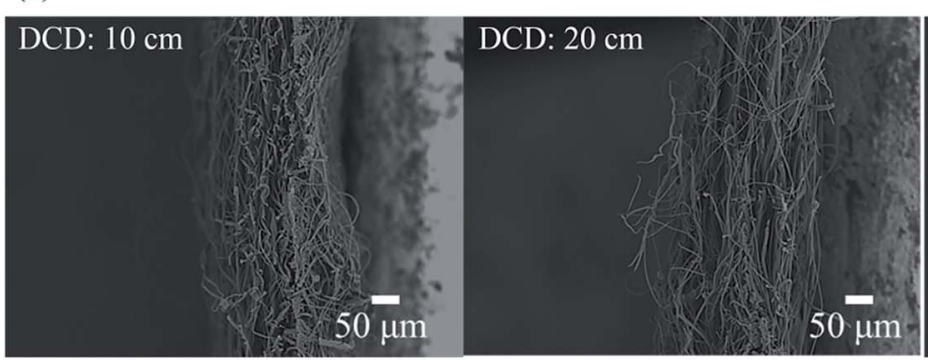

Compact structure (b)

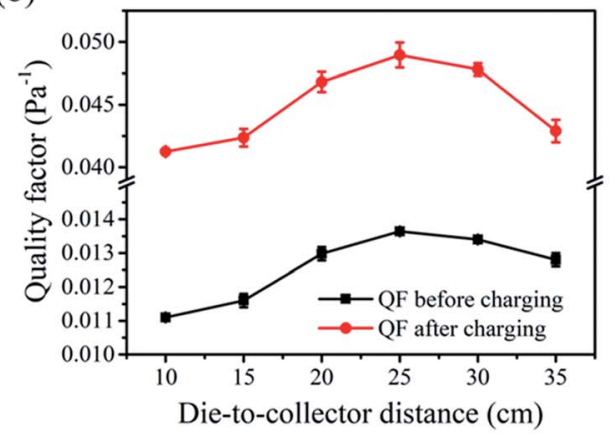


structure severely reduced the filtration efficiency. Based on these results, we obtained the benefit-to-cost of DCD $(25 \mathrm{~cm})$ and further confirmed the key role of a fluffy structure in improving the filtration performance.

In order to improve the filtration performance, corona charging technology was adopted and the filtration performances of the samples after charging were studied. As illustrated in Fig. 3a, the filtration efficiencies of the charged samples were 93.68\%, 93.10\%, 92.81\%, 92.57\%, 90.96\% and $87.56 \%$, while the pressure drops were $67.3,63.1,56.3$, 53.1, 50 and $48.6 \mathrm{~Pa}$, respectively. As compared to the uncharged samples, the filtration efficiency of the charged samples displayed a similar trend with an increase in DCD except that the filtration efficiency registered a remarkable improvement. This was primarily due to the fact that the charged fibers possess strong electrostatic force that can capture particles by electrostatic attraction. ${ }^{28}$ Moreover, the pressure drop remained almost the same, which can be ascribed to the fact that corona charging does not transform the structures of melt blown nonwovens. Moreover, the QF value of the samples after charging significantly improved and displayed a similar trend with the value before charging, which further confirms that the contribution of electrostatic force improves the filtration efficiency without sacrificing the pressure drop.

\section{Design and fabrication the PP/MgSt melt blown nonwovens}

The web properties. It is well known that pressure drop and filtration efficiency are two important factors for filtration materials. Since we have already fabricated a fluffy melt blown material with low pressure drop, to further improve the filtration performance, MgSt, a charge enhancer that can enhance the electret performance, was introduced to the PP polymer. The web morphologies were first investigated. As shown in Fig. 4a-f, no visible difference in fiber diameter was found when compared to the pure PP sample (Fig. $4 \mathrm{~g}$ and $\mathrm{S} 3 \dagger$ ). Moreover, the PP/MgSt samples presented smooth surfaces with no agglomerates. This result can be explained by the fact that the MgSt particles melted at high temperature and thus uniformly dispersed in the PP fluid. In our study, the MgSt particles were added together with PP chips into the extruder during the melt blown process. Since the melting point of MgSt is about $200{ }^{\circ} \mathrm{C}$ and the extruder temperature is higher than $282{ }^{\circ} \mathrm{C}$, the $\mathrm{MgSt}$ particles would melt during this process. Compared to inorganic particles, such as barium titanate, ${ }^{29}$ tourmaline $^{\mathbf{1 4}}$ and $\mathrm{TiO}_{2}$ particles, ${ }^{30}$ which would aggregate on the fiber surface, the MgSt particles melt mixed with the PP fusant and no agglomerates were observed. Furthermore, the porosity, average pore size and pore size distribution were investigated. As shown in Fig. $4 \mathrm{~h}$, the average pore size of the samples ranged from 14.2
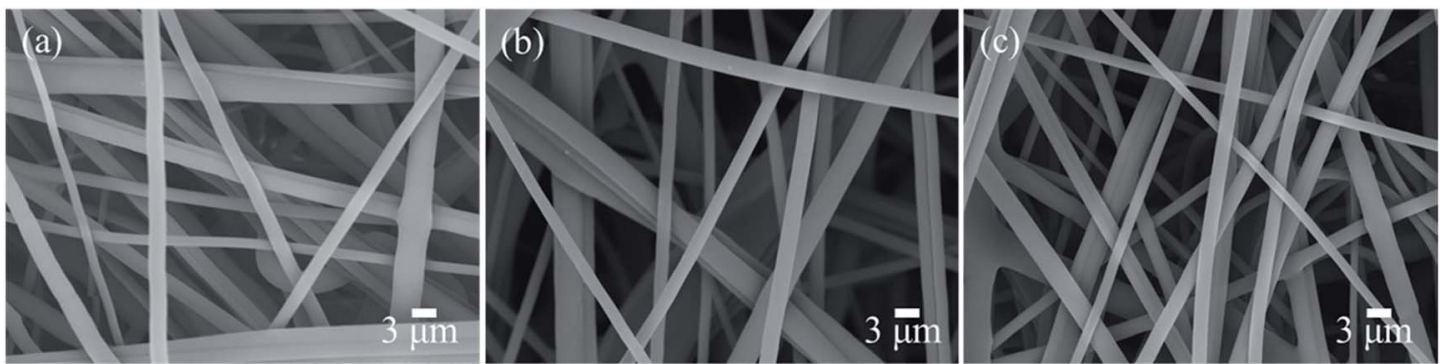

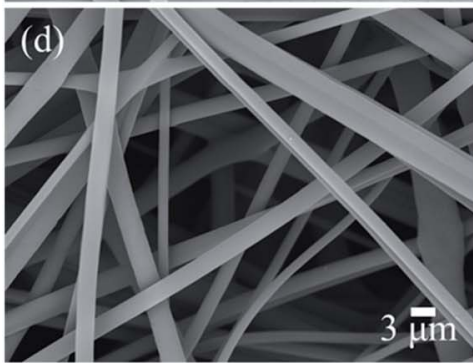

(g)

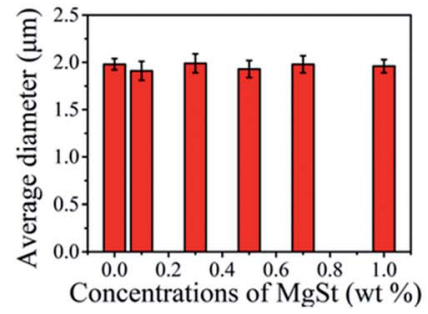

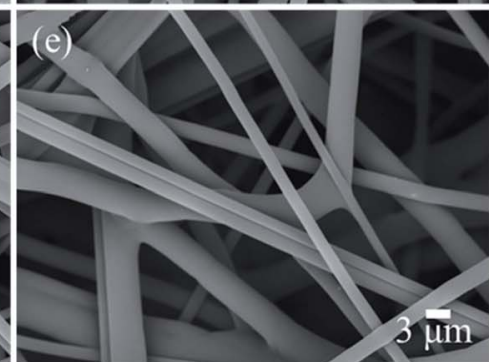

(h)

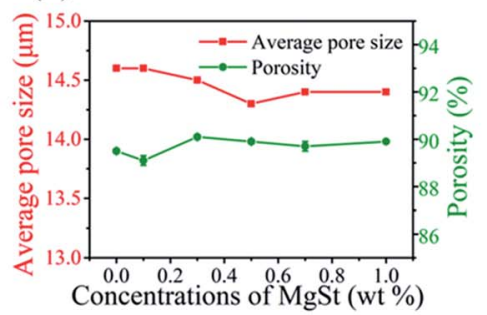

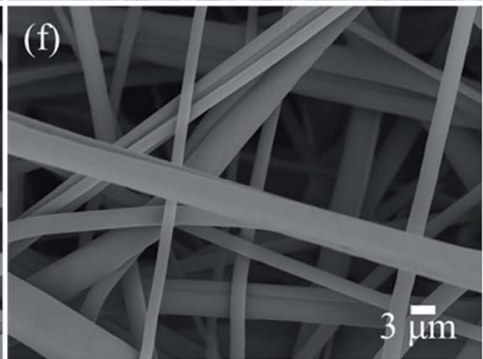

(i)

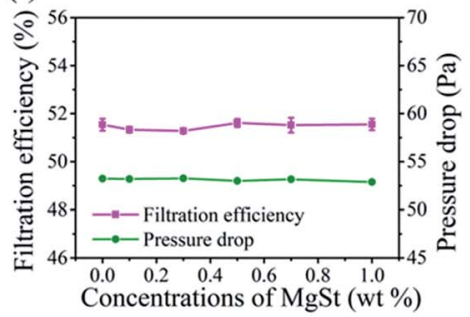

Fig. 4 Morphology, pore structure and filtration efficiency of PP/MgSt melt blown nonwovens. SEM images of PP melt blown containing MgSt particle concentrations of (a) 0, (b) 0.1, (c) 0.3, (d) 0.5, (e) 0.7 and (f) 1 wt\%. (g) Average diameter, (h) average pore size and porosity, (i) filtration performance of the uncharged samples. 
$\mu \mathrm{m}$ to $14.6 \mu \mathrm{m}$ and the porosity ranged from $88.7 \%$ to $90.1 \%$. In addition, there was no visible difference in the pore size distributions (Fig. S4†). From the foregoing fiber and pore structure results, we can conclude that the morphologies of the nonwovens did not change after the introduction of the additives. We further investigated the filtration performances of the samples containing various concentrations of MgSt before charging, which is shown in Fig. 4i. As expected, both the filtration efficiency and the pressure drop are similar with an increase in MgSt concentrations from 0 to $1 \mathrm{wt} \%$, confirming that the MgSt does not influence the filtration performance achieved by mechanical interception.

The filtration and electret properties. The corona charging technology was then adopted to improve the filtration efficiency by the electrostatic capture mechanism. As demonstrated in Fig. 5a, the filtration efficiency of the charged PP sample was 92.57\% and the pressure drop was 53.1 Pa. Compared to the PP sample, the PP/MgSt samples exhibited higher filtration efficiency and with an increase in MgSt amount to $0.5 \mathrm{wt} \%$, the samples achieved a filtration efficiency of $95.90 \%$ with a pressure drop of $52.9 \mathrm{~Pa}$. This clearly demonstrated that MgSt can improve the filtration performance, which can be explained by more particle collection from the electrostatic force. ${ }^{31}$ Nevertheless, with further increase in amount to $1 \mathrm{wt} \%$, the filtration efficiency showed a steady state value of $95.89 \%$, suggesting that there is a saturation value for the MgSt content. QF values of the samples having MgSt particles were calculated (Fig. 5b). QF of the samples containing MgSt presented an escalating trend with an increase in MgSt concentrations and then attained a constant state.

The stability of filtration efficiency was a great property for the electret filtration materials duo to the charges would attenuate after a period of time, which would result in the decline of filtration efficiency. Therefore, an accelerated decay experiment was conducted at high temperature to investigate the filtration efficiency stability. As shown in Fig. 6a, after heat treatment at $70{ }^{\circ} \mathrm{C}$ for $24 \mathrm{~h}$, the filtration efficiency of the pure PP sample descended from $92.57 \%$ to $90.97 \%$ (by $1.60 \%$ ), while the decrements of the samples containing various concentrations MgSt were $1.50 \%, 1.40 \%, 0.48 \% 0.42$ and $0.40 \%$. Clearly, the PP/MgSt samples showed better stability of filtration efficiency and with an increase in the MgSt concentrations, the decay exhibited a significantly decreasing trend, demonstrating that MgSt is a fine additive to improve the filtration performance stability. In addition, when MgSt concentrations exceed $0.5 \mathrm{wt} \%$, the decay is nearly constant.

To uncover the mechanism of such decay, a surface voltage decay experiment was performed. As illustrated in Fig. S5, $\dagger$ after heat treatment, the surface voltage plots displayed similar decaying trend with the filtration efficiency, confirming that the decay of surface voltage was the main reason of the decrease in filtration efficiency. In addition, with the increase of MgSt concentrations, the decay of the surface potential reduced, indicating that samples containing MgSt possessed superior stability of charge, which was mainly due to the fact that more charges were captured in deeper traps and thus could not easily (a)

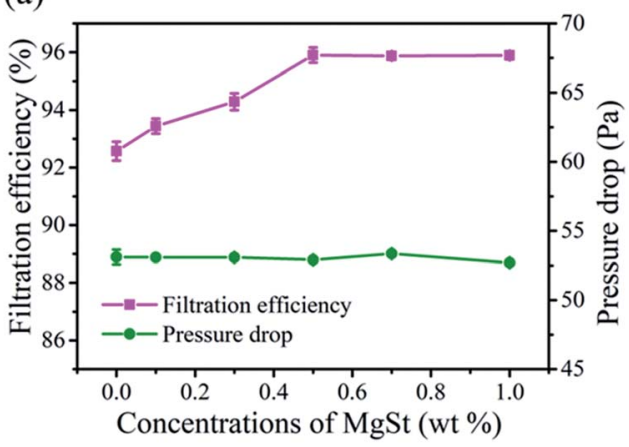

(b)

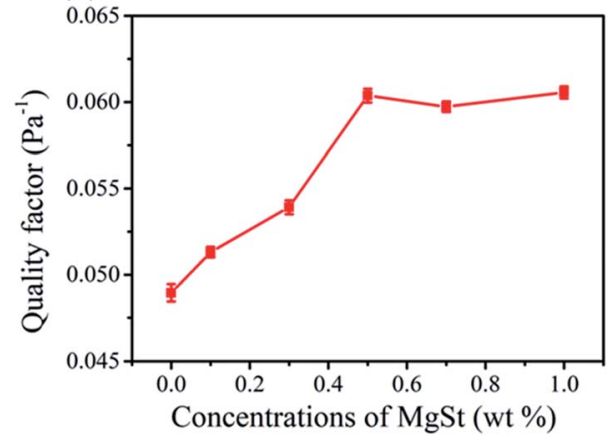

Fig. 5 Filtration performance of the charged PP/MgSt melt blown nonwovens. (a) Filtration efficiency and pressure drop and (b) quality factor value. (a)

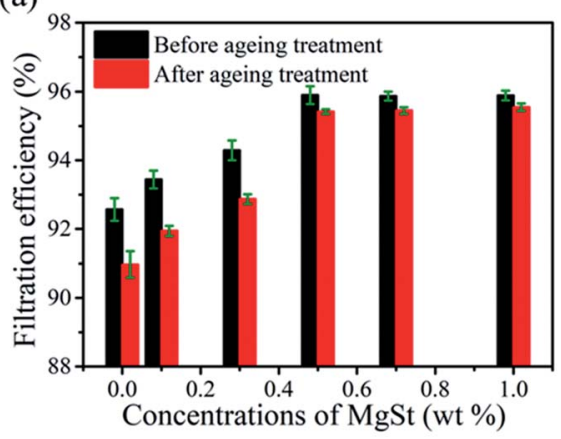

(b)

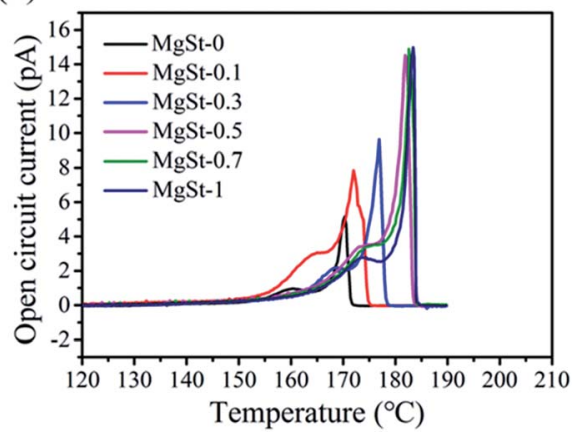

Fig. 6 The stability of filtration and electret performance. (a) The filtration efficiency stability of PP/MgSt samples and (b) the TSD spectra of the PP/MgSt melt blown nonwovens. 
escape. ${ }^{17}$ TSD current measurements were also used to investigate the charge trap parameters and the phase transition of the polymer because of the high sensitivity of charge trap parameters to the structure of polymers. ${ }^{32}$ The TSD spectra of the samples are shown in Fig. 6b. It can be observed that the shape of the peaks is similar, while both the peak intensity and the corresponding temperature are different. In addition, there are two peaks for all of the samples, which indicates that charges are primarily captured in two types of trap energy bands that are deep and shallow. ${ }^{33}$ Furthermore, for the pure PP sample, the main peak appeared at about $170{ }^{\circ} \mathrm{C}$, while the peaks of samples containing $0.1 \mathrm{wt} \%, 0.3 \mathrm{wt} \%, 0.5 \mathrm{wt} \%, 0.7 \mathrm{wt} \%$ and $1 \mathrm{wt} \%$ concentrations of $\mathrm{MgSt}$ appeared at $172{ }^{\circ} \mathrm{C}, 177{ }^{\circ} \mathrm{C}, 182{ }^{\circ} \mathrm{C}$, $182{ }^{\circ} \mathrm{C}$ and $183{ }^{\circ} \mathrm{C}$, respectively. In contrast, the main peak of the $\mathrm{PP} / \mathrm{MgSt}$ sample shifted to a higher temperature and the slight shift indicated that the trap energy bands changed to a high band. ${ }^{34}$ This result revealed that MgSt is beneficial to the charge storage stability. In particular, we found that the peak intensity significantly increased with an increase in $\mathrm{MgSt}$ concentrations from 0 to $0.5 \mathrm{wt} \%$, which confirms that more charges were trapped in the $0.5 \mathrm{wt} \% \mathrm{MgSt}$ sample.

The mechanism of improving the electret properties. To reveal the mechanism for how the MgSt particles improve the electret performance, the crystal structures were investigated and the crystal parameters were calculated. The XRD patterns are shown in Fig. 7a and other crystal parameters are listed in Table S1. $\dagger$ It can be observed that the lattice plane (110) appears at $14.1^{\circ}, 14.0^{\circ}, 14.3^{\circ}, 14.1^{\circ}, 14.1^{\circ}$ and $14.1^{\circ}$. This is in good agreement with the $\alpha$-crystals. Therefore, we speculate that the fibers were predominately crystallized into $\alpha$-crystals. The crystallinity was also calculated. The crystallinity is $27.1 \%$ for the pure PP sample, while the crystallinity of the samples containing $0.1 \mathrm{wt} \%, 0.3 \mathrm{wt} \%, 0.5 \mathrm{wt} \%, 0.7 \mathrm{wt} \%$ and $1 \mathrm{wt} \%$ concentration of $\mathrm{MgSt}$ are $31.6 \%, 37.6 \%, 45.2 \%, 45.6$ and $46.1 \%$, respectively (Fig. 7b). These results indicate that MgSt can improve crystallinity. In addition, for the PP/MgSt samples, the lattice plane (110) thicknesses ranged from $66 \AA$ to $99 \AA$, which are smaller than those of the pure PP sample (100 $\AA$ ), suggesting that more fine-grained morphology was formed owing to the addition of MgSt. As illustrated in Fig. 7c, for the pure PP material, the crystal nucleus is generated by molecular motion, and the nucleation rate is slow, which results in a large spherical size. For the samples with nucleating agents, however, because of the presence of vast heterogeneous nucleation, the spherulites would collide with each other and there was no time for the spherulites to grow. On this basis, the mechanism of improving the electret properties can be explained by the change of the crystal structure caused by the addition of MgSt particles. On one hand, the charge quantity increased primarily because for the semi-crystalline materials, similar to PP, the (a)

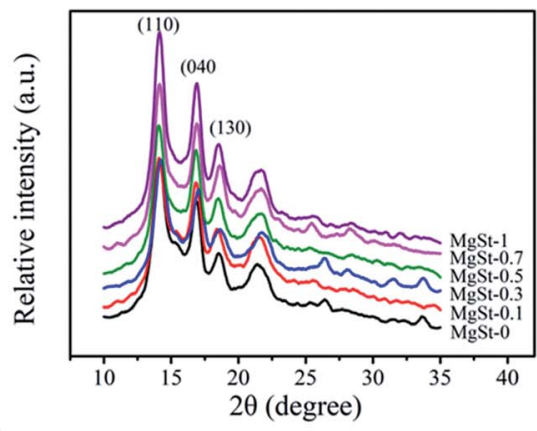

(c)

Polymer melt
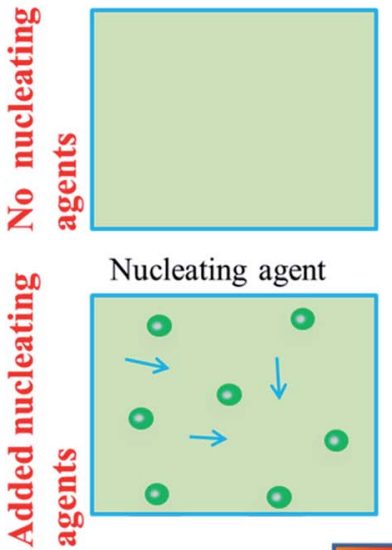

(b)

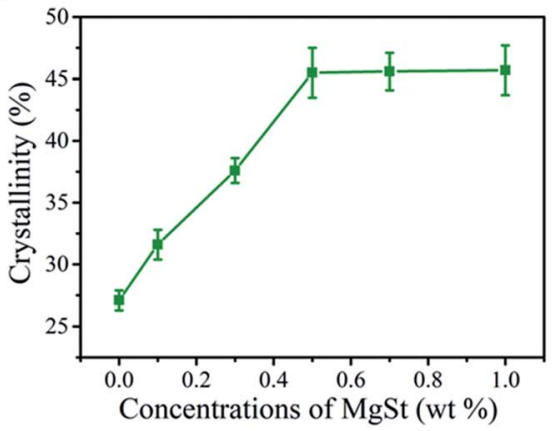

Partly crystallize

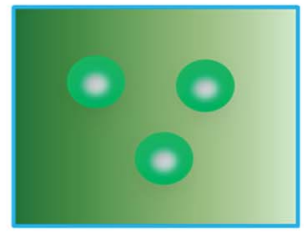

Totally crystallize

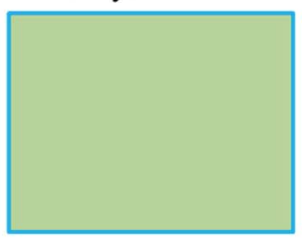

Starting crystallization

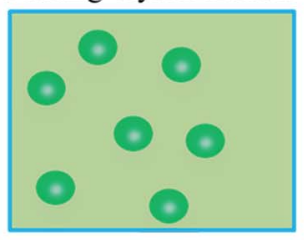

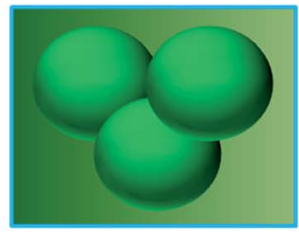

Totally crystallize
Partly crystallize

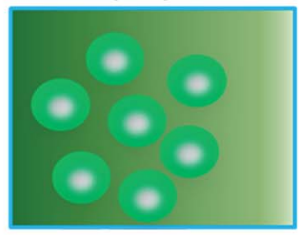

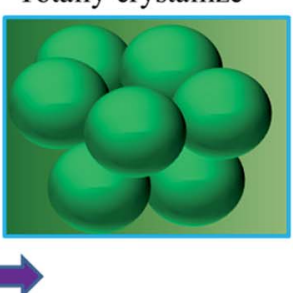

Cooling

Fig. 7 The crystal structures of the PP/MgSt melt blown nonwovens. (a) XRD diffraction pattern of PP/MgSt nonwovens, (b) the crystallinity of the PP/MgSt nonwovens, (c) the nucleation processes for the pure PP melt blown nonwoven and the PP/MgSt samples. 
concentration and nature of traps are expected to depend on factors such as amorphous-to-crystalline boundaries, defect sites, and impurities..$^{32}$ In this study, the samples containing MgSt particles produced larger crystal-amorphous boundaries, where space charges were located. On the other hand, the charge stability improved because the shallow traps primarily exist in the boundaries and peripheral regions, while the deep traps are located in the central parts. The ratio of deep traps to shallow traps increased with the decrease in spherulite size. ${ }^{35}$

Evaluation of filtration performance. The filtration efficiency can be further improved by regulating the basis weights. As shown in Fig. 8a, with an increase in basis weights, the filtration efficiency and pressure drop of the $\mathrm{PP} / \mathrm{MgSt}$ melt blown nonwoven increased gradually. The filtration efficiency reached a steady value when the basis weight was $40 \mathrm{~g} \mathrm{~m}^{-2}$, while the pressure drop presented a quasi-linear growth tendency with a slope of 2 . This indicated that the efficiency can be improved by enhancing the basis weight, primarily because the increased numbers of fibers may increase tortuous airflow channels and thus, the particles are more easily captured by mechanical interception. ${ }^{36}$ It is interesting to notice that the personal respirator standard (filtration efficiency $\geq 99 \%$ and a pressure drop $\leq 120 \mathrm{~Pa}$ ) could be met by increasing the basis weight to $40 \mathrm{~g} \mathrm{~m}^{-2}$. Therefore, the PP/MgSt melt blown nonwoven with a basis weight of $40 \mathrm{~g} \mathrm{~m}^{-2}$ was further studied.

The filtration efficiency of the PP/MgSt nonwovens under various air flow rates $\left(30-80 \mathrm{~L} \mathrm{~min}^{-1}\right)$ were systematically studied (Fig. 8b). A commercial melt blown nonwoven and an (a)

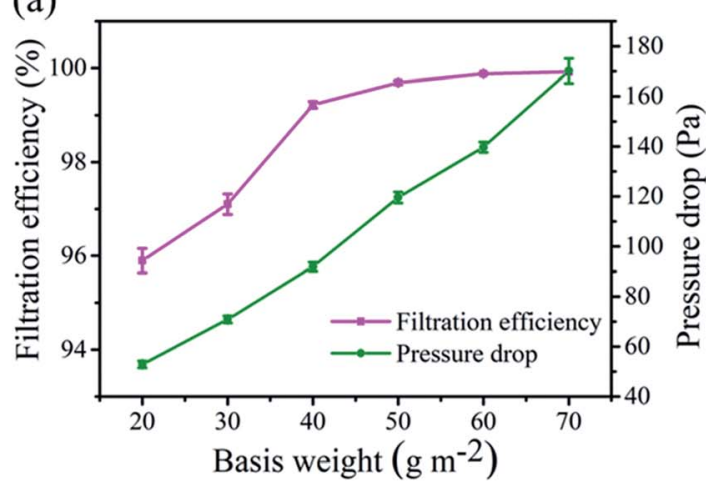

(c)

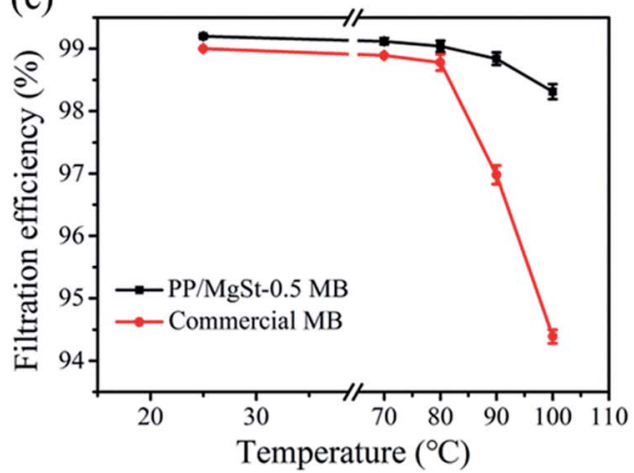

(e)

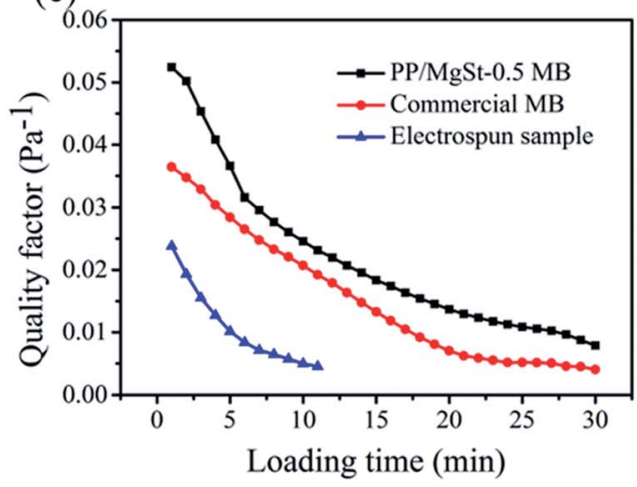

(b)

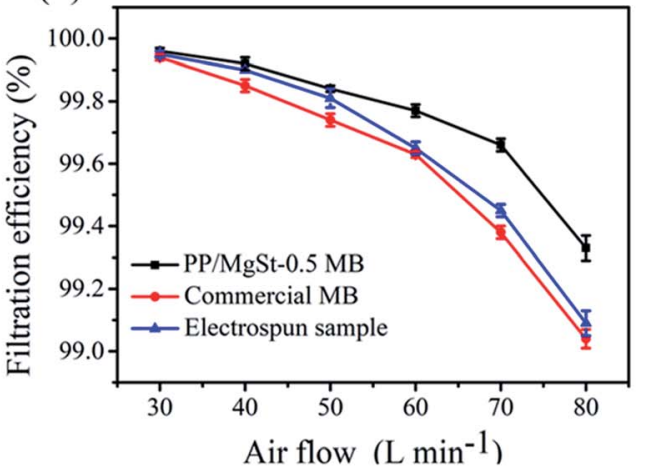

(d)

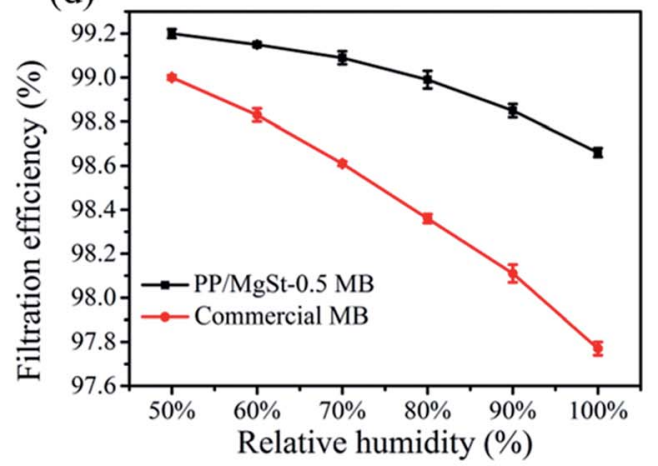

(f)

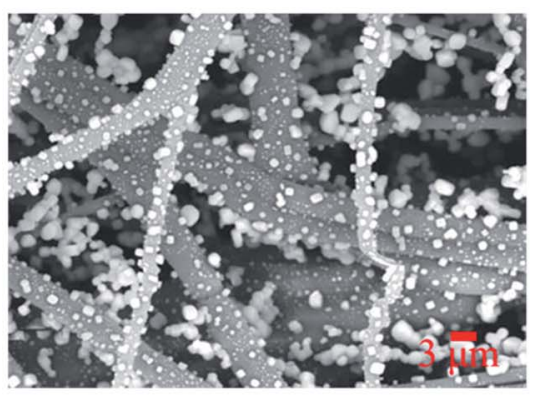

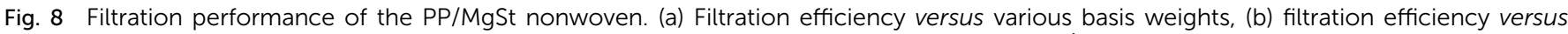

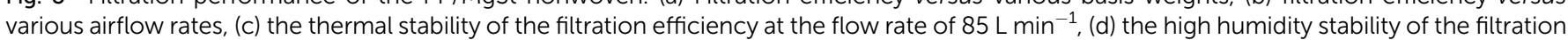

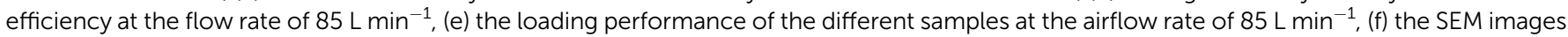
of the PP/MgSt samples after the loading filtration test. 
electrospun membrane were also investigated for comparison. Their basic characteristics are listed in Table S2. $\uparrow$ The PP/MgSt sample showed the best filtration performance $(99.33 \%$ at the rate of $80 \mathrm{~L} \mathrm{~min}^{-1}$ ) among all the three samples. However, the commercial melt blown sample and the electrospun membrane showed a faster decline of filtration efficiency with the increase in flow rate. This result could be explained by the fact that the electrospun nanofibers primarily filtrated the particles through their fine fiber diameter, small pore size and large surface area to volume ratio. ${ }^{37}$ When the airflow increased, the retention time of particles in the samples reduced, which directly reduced the possibility for particles to collide on the fibers through Brownian diffusion. ${ }^{38}$ Nevertheless, for the electret melt blown nonwovens, the particles were captured not only by the mechanical effect but also by electrostatic attraction. When the airflow increased, the powerful electrostatic force could also help capture the particles in a short time.

Considering that the filtration stability is a very important performance parameter during the storage procedure in practical applications, the thermal and humidity stability of the filtration performance was further investigated. As shown in Fig. 8c, the PP/MgSt sample presented prominent thermal stability with a gentle decrease in filtration from $99.22 \%$ to $98.41 \%$ after placing it at $100{ }^{\circ} \mathrm{C}$ for $24 \mathrm{~h}$. In contrast, the commercial melt blown samples showed a sharp decrease in filtration efficiency when treated at $90{ }^{\circ} \mathrm{C}$ for $24 \mathrm{~h}$. Similarly, with an increase in relative humidity, both the PP/MgSt sample and the commercial sample showed a gradually decaying tendency, but the PP/MgSt maintained a high filtration efficiency of $98.66 \%$, which was higher than the commercial sample (97.77\%) after treating it at a high RH of $100 \%$ (Fig. $8 d$ ). These results demonstrate that the $\mathrm{PP} / \mathrm{MgSt}$ sample possess better stability than the commercial sample.

The dynamic filtration behavior is shown in Fig. 8e. QF values for all the three samples decreases when the loading time increases. Among them, the electrospun membrane showed the lowest $Q F$ value and reached the minimum value within $12 \mathrm{~min}$. In contrast, the PP/MgSt sample and the commercial sample maintained higher QFs, which became constant after loading for $20 \mathrm{~min}$. This indicated that the melt blown nonwovens possess better dynamic filtration behavior. During the loading test, particles deposited on both the surfaces and interiors of the samples (Fig. 8f), leading to the formation of a dendrite structure inside and caused the gradual closing of the open channels. $^{39}$ The nanometer-scaled structure easily blocked the electrospun membrane; therefore, the pressure drop rose rapidly. Benefiting from the fluffy structure with larger porosity, the pressure drop of the PP/MgSt sample increased slower than the commercial sample (Fig. S6 $\dagger$ ). These results suggest that the melt blown nonwovens have better dust containing capacity and a longer service life.

\section{Conclusions}

In summary, we successfully designed a fluffy PP/MgSt electret melt blown filtration material with high filtration efficiency and low air resistance. First, a fluffy nonwoven filter with fine fiber diameter, small pore size and high porosity was achieved by regulating the melt blown DCD. Then, by introducing a nucleating agent into the PP polymer during the melt blown process, the electret performance of the resultant nonwoven filter improved significantly. The filtration performance revealed that the nonwoven possessed high filtration efficiency up to $99.22 \%$, low pressure drop of $92 \mathrm{~Pa}$ and satisfactory QF value of 0.054 $\mathrm{Pa}^{-1}$ at an air flow rate of $85 \mathrm{~L} \mathrm{~min}^{-1}$ and particle mass median diameter of $0.26 \mu \mathrm{m}$. This value is far superior to the current personal respirator standard. In particular, this material exhibited excellent electret stability, which guarantees its longterm storage and usage. Collectively, we anticipate that the melt blown electret material developed in this study is a good candidate for PM2.5 filtration.

\section{Conflicts of interest}

The authors declared no potential conflicts of interest with respect to the research, authorship and/or publication of this article.

\section{Acknowledgements}

This work is supported by the Key Technologies R \& D Program of China (2015BAE01B01).

\section{Notes and references}

1 C. Liu, P. Hsu, H. Lee, M. Ye, G. Zheng, N. Liu, W. Li and Y. Cui, Nat. Commun., 2015, 6, 6205.

2 X. Zhao, Y. Li, T. Hua, P. Jiang, X. Yin, J. Yu and B. Ding, ACS Appl. Mater. Interfaces, 2017, 9, 12054-12063.

3 G. Mouret, D. Thomas, S. Chazelet, J. Appert-Collin and D. Bemer, J. Aerosol Sci., 2009, 40, 762-775.

4 S. R. Ardkapan, M. S. Johnson, S. Yazdi, A. Afshari and N. C. Bergsøe, J. Aerosol Sci., 2014, 14-20.

5 R. S. Barhate and S. Ramakrishna, J. Membr. Sci., 2007, 296, 1-8.

6 S. Zhang, H. Liu, J. Yu, W. Luo and B. Ding, J. Mater. Chem. A, 2016, 4, 6149-6157.

7 R. S. Barhate, C. K. Loong and S. Ramakrishna, J. Membr. Sci., 2006, 283, 209-218.

8 B. Y. Yeom, E. Shim and B. Pourdeyhimi, Macromol. Res., 2010, 18, 884-890.

9 M. A. Hassan, B. Y. Yeom, A. Wilkie, B. Pourdeyhimi and S. A. Khan, J. Membr. Sci., 2013, 427, 336-344.

10 R. Uppal, G. Bhat, C. Eash and K. Akato, Fibers Polym., 2013, 14, 660-668.

11 G. Chen, H. Xiao and X. Wang, in Properties and Applications of Dielectric Materials. ICPADM 2009, IEEE 9th International Conference on the (IEEE, 2009), 2009, pp. 389-391.

12 R. Thakur, D. Das and A. Das, Sep. Purif. Rev., 2013, 42, 87129.

13 R. Thakur, D. Das and A. Das, Fibers Polym., 2014, 15, 14361443. 
14 B. Yu, J. Han, H. Sun, F. Zhu, Q. Zhang and J. Kong, Polym. Compos., 2014, 36, 264-271.

15 A. Kilic, E. Shim and B. Pourdeyhimi, Aerosol Sci. Technol., 2015, 49, 666-673.

16 A. Brochocka, K. Majchrzycka and K. Makowski, Fibres Text. East. Eur., 2013, 21, 100.

17 H. Xiao, J. Gui, G. Chen and C. Xiao, J. Appl. Polym. Sci., 2015, 132, 42807-42812.

18 Y. Yesil and G. S. Bhat, J. Text. Inst., 2017, 108, 1035-1040.

19 J. Feng, Mater. Lett., 2017, 189, 180-183.

20 R. Thakur, D. Das and A. Das, J. Text. Inst., 2013, 1-9.

21 X. M. Wang and Q. M. Zhao, Polym. Eng. Sci., 2008, 48, 21432146.

22 V. Bansal, Ind. Eng. Chem. Res., 1998, 37, 1799-1806.

23 Y. Yesil, Y. Yesil, G. S. Bhat and G. S. Bhat, Int. J. Cloth. Sci. Tech., 2016, 28, 780-793.

24 Y. C. Zeng and X. H. Wang, J. Appl. Polym. Sci., 2011, 119, 2112-2123.

25 Y. Yang, S. Zhang, X. Zhao, J. Yu and B. Ding, Sep. Purif. Technol., 2015, 152, 14-22.

26 S. Zhang, H. Liu, X. Yin, J. Yu and B. Ding, ACS Appl. Mater. Interfaces, 2016, 8, 8086-8095.

27 C. Hung and W. W. Leung, Sep. Purif. Technol., 2011, 79, 3442.
28 J. H. Ji, G. N. Bae, S. H. Kang and J. Hwang, J. Aerosol Sci., 2003, 34, 1493-1504.

29 A. A. Guzhova, M. F. Galikhanov, Y. A. Gorokhovatsky, D. E. Temnov, E. E. Fomicheva, E. A. Karulina and T. A. Yovcheva, J. Electrost., 2016, 79, 1-6.

30 A. Viraneva, T. Yovcheva, I. Bodurov and M. Galikhanov, Bulg. Chem. Commun., 2013, 45, 73-76.

31 C. Wang, Powder Technol., 2001, 118, 166-170.

32 R. Nath and M. M. Perlman, IEEE Trans. Electr. Insul., 1989, 24, 409-412.

33 M. Tang, Z. An, Z. Xia and X. Zhang, J. Electrost., 2007, 65, 203-208.

34 Z. An, J. Yao, M. Mao, Y. Zhang and Z. Xia, J. Electrost., 2010, 68, 523-527.

35 T. Yamashita and K. Ikezaki, J. Electrost., 2005, 63, 559-564. 36 N. Wang, Y. Yang, S. S. Al-Deyab, M. El-Newehy, J. Yu and B. Ding, J. Mater. Chem. A, 2015, 3, 23946-23954.

37 F. E. Ahmed, B. S. Lalia and R. Hashaikeh, Desalination, 2015, 356, 15-30.

38 D. C. Walsh and J. Stenhouse, J. Aerosol Sci., 1997, 28, 307321.

39 K. Li and Y. M. Jo, Aerosol Sci. Technol., 2010, 44, 578-587. 